\title{
In Vitro Virucidal Effect of Intranasally Delivered Chlorpheniramine Maleate Compound Against Severe Acute Respiratory Syndrome Coronavirus 2 (SARS-CoV-2)
}

\author{
Authors: Jonna Westover, $\mathrm{PhD}^{1}$; Gustavo Ferrer, $\mathrm{MD}^{2}$ \\ 1. Utah State University, Institute for Antiviral Research \\ 2. Ferrer Medical Innovations. Nova Southeastern University
}

\begin{abstract}
Background. The initial global outbreak of the novel coronavirus disease 2019 (COVID-2019) pandemic, responsible for the severe acute respiratory syndrome 2 (SARS-CoV-2), was first reported in Wuhan, China, at the end of December 2019. COVID-19 shares similarities with the severe acute respiratory syndrome coronavirus (SARS-CoV), Middle East respiratory syndrome coronavirus (MERS-CoV), and it behaves similarly to influenza with a high intranasal viral load. The genome sequence of COVID-19 opened the opportunity for multiple in vitro and clinical trials, but we still do not have a clear path to treatment. Chlorpheniramine is a safe and effective antihistamine with potent antiviral activity against various strains of influenza $\mathrm{A} / \mathrm{B}$, thus suggesting that CPM has broad antiviral activity. We tested the virucidal potential of chlorpheniramine maleate (CPM) in a nasal spray composition currently in development as an anti-allergy medication.
\end{abstract}

Methods. The virucidal activity of chlorpheniramine maleate was tested using viral stock of SARS-CoV-2, USA-WA1/2020 strain in Vero 76 infected cells. The end-point titer (CCID50) values were calculated with the Reed-Muench (1948) equation. Three independent replicates of each sample were tested, and the average and standard deviation were calculated. Results were compared with untreated controls by one-way ANOVA with Dunnett's multiple comparison test in GraphPad Prism (version 8) software. 
Results. After 25-minutes of contact time, the nasal spray reduced the levels of the virus from 4.2 to $1.7 \log 10$ CCID50 per $0.1 \mathrm{~mL}$, a statistically significant reduction of $2.5 \log 10$ CCID50.

Conclusions. This study demonstrates the strong virucidal effect against SARS-CoV-2 of a nasal spray containing chlorpheniramine maleate. Given that CPM has broad antiviral effects against influenza, virucidal effect against SARS-CoV-2, and coadjuvant effects with hydroxychloroquine in treating multidrug-resistant malaria with minimal side effects. We propose two further studies: a randomized placebo-controlled study of intranasally delivered chlorpheniramine in patients with mild to moderate SARS-CoV-2, and a second study aiming to determine the potential antiviral and adjuvant effects of CPM plus hydroxychloroquine, versus chloroquine alone, in hospitalized patients with SARS-CoV-2.

\section{INTRODUCTION}

The initial global outbreak of the novel coronavirus disease 2019 (COVID-2019) pandemic, responsible for the severe acute respiratory syndrome 2 (SARS-CoV-2), was first reported in Wuhan, China, at the at the end of December 2019․ As of April 16, 2020, there were more than 2 million infected cases and more than 144,000 deaths in more than 200 countries. SARS-CoV-2 shares similarities with severe acute respiratory syndrome coronavirus (SARS-CoV) and Middle East respiratory syndrome coronavirus (MERS-CoV). The genomic sequence of SARS-CoV-2 has enabled multiple in vitro and clinical trials aiming to help infected patients. Unfortunately, evidence supporting any of the proposed prophylactic or treatments is negligible.

In an attempt to repurpose existing drugs with antiviral potential that might be used in COVID19 prevention and treatment, we tested the virucidal potential of chlorpheniramine maleate (CPM) in a nasal spray composition currently in development as an anti-allergy medication. CPM is a first-generation antihistamine that has been marketed in the United States for several decades and is currently widely used. Recently published literature suggests that CPM not only is effective in treating anaphylaxis but also has strong antiviral and anti-inflammatory activity. Most notably, a 2018 article highlighted the substance's antiviral activity in various strains of influenza $\mathrm{A} / \mathrm{B}$, thus suggesting that it may have greater activity than the FDA-approved 
Tamiflu3 . Moreover, CPM was found to inhibit infection by other influenza A viruses, specifically 2009(H1N1), 1934(H1N1), and 1989(H3N2) strains, as well as an influenza B virus, B/Shanghai/2017(BY), thus suggesting that CPM has broad antiviral activity ${ }^{3}$.

Moreover, multiple in vitro studies have demonstrated that CPM reverses chloroquine resistance against an African isolate of $P$. falciparum ${ }^{4,5}$. In a clinical study, chlorpheniramine has been found to enhance the efficacy of chloroquine in treating acute uncomplicated $P$. falciparum infections in children from an endemic area of Nigeria ${ }^{6}$. In a study by Sowunmi et al., CPM at higher dosages has been found to produce a more significant and beneficial action ${ }^{7}$. Moreover, an in vitro study has demonstrated that chlorpheniramine has synergistic effects with chloroquine, mefloquine, quinine or pyronaridine against both multidrug-resistant or chloroquine-resistant P. falciparum ${ }^{8,9}$.

Chlorpheniramine is a safe and effective antihistamine whose main side effect is drowsiness. However, evidence suggests that intranasal delivery shows high efficacy with no side effects. An animal study of intranasally delivered CPM has shown that it does cross into the brain circulation ${ }^{10}$. Van Toor et al. have assessed the systemic bioavailability and safety of a nasal spray solution developed to deliver doses of 1.12 and $2.24 \mathrm{mg}$ CPM intranasally ( $0.4 \%$ nasal spray) and have found no adverse events ${ }^{11}$. In another study, Kirkegaard et al. have found that intranasally delivery of CPM significantly inhibits histamine-induced symptoms ${ }^{12}$.

Given the strong evidence supporting CPM's antiviral, anti-inflammatory and possible coadjuvant effects with hydroxychloroquine, a drug currently used to treat COVID-19, we performed an in vitro study to test the virucidal effect of nasal spray containing CPM as an active ingredient in addition to other excipients 


\section{METHODS}

\section{Nasal Spray Composition}

Table 1 Nasal Spray Composition

\begin{tabular}{|c|}
\hline CoMPONENT \\
\hline Purified Water \\
\hline Chlorpheniramine Maleate \\
\hline Xylitol \\
\hline Glycerin \\
\hline Sodium Bicarbonate \\
\hline
\end{tabular}

* Nasal spray composite with CPM at $0.4 \%--3.6 \mathrm{mg} / \mathrm{mL}$

**Nasal spray composition developed by Ferrer Medical Innovations and formulated by Xlear, Inc ***Stability testing performed at three months (see appendix A)

\section{Procedure}

\section{Virus, media and cells}

The viral stock of SARS-CoV-2, USA-WA1/2020 strain, was prepared before testing through growth in Vero 76 cells. The culture medium for the prepared stock (test medium) was MEM with $2 \%$ fetal bovine serum and $50 \mu \mathrm{g} / \mathrm{mL}$ gentamicin.

\section{Virucidal assay}

Nasal spray was provided by the sponsor. The compound was mixed directly with virus solution at a proportion of $90 \%$ compound preparation and $10 \%$ virus solution. A single concentration was tested in triplicate. Test medium without virus was added to one tube of the prepared compound to serve as a toxicity control. Ethanol (70\%) was tested in parallel as a positive control, and water only was tested as a virus control. The solution and virus were incubated at 
room temperature $\left(22 \pm 2^{\circ} \mathrm{C}\right)$ for 25 minutes. The solution was then neutralized through $1 / 10$ dilution in test medium.

\section{Viral quantification}

Surviving virus from each sample was quantified with standard end-point dilution assays. Briefly, samples were serially diluted $1 / 10$ in test medium. Then $100 \mu \mathrm{L}$ of each dilution was plated into quadruplicate wells of 96-well plates containing 80-90\% confluent Vero 76 cells. the plates were incubated at $37 \pm 2^{\circ} \mathrm{C}$ under $5 \% \mathrm{CO}_{2}$ for 6 days. Each well was then scored for the presence or absence of virus. The end-point titer (CCID50) values were calculated with the Reed-Muench (1948) equation.

\section{Statistical analysis}

Three independent replicates of each sample were tested, and the average and standard deviation were calculated. Results were compared with untreated controls by one-way ANOVA with Dunnett's multiple comparison test in GraphPad Prism (version 8) software.

Controls: Virus controls were tested in water, and the reduction of virus in the test wells compared with the virus control wells was calculated as the log reduction value (LRV). Toxicity controls were tested with medium not containing virus to determine whether the samples were toxic to cells. Neutralization controls were tested to ensure that viral inactivation did not continue after the specified contact time and that any residual sample in the titer assay plates did not inhibit growth and detection of surviving virus. We performed this procedure by adding toxicity samples to titer test plates, then spiking each well with a small amount of virus that would produce an observable CPE during the incubation period. 
Table 2. Virucidal efficacy of nasal spray against SARS-CoV-2 after a 25-minute incubation with virus at $22 \pm 2{ }^{\circ} \mathrm{C}$.

\begin{tabular}{lcccc}
\hline & Concentration & Incubation & Virus Titer $^{\mathrm{a}, \mathrm{c}}$ & LRV $^{\mathrm{b}}$ \\
\hline Nasal spray & $90 \%$ & 25 -minutes & $1.7 \pm 0.0$ & $2.5^{* * *}$ \\
Ethanol & $67.5 \%$ & 25 -minutes & $1.0 \pm 0.6$ & $3.2^{* * *}$ \\
Virus Control & na & 25 -minutes & $4.2 \pm 0.4$ & na \\
& & & & \\
\hline
\end{tabular}

${ }^{a} \log 10$ CCID50 of virus per $0.1 \mathrm{~mL}$, average of three replicates \pm standard deviation

${ }^{\mathrm{b}} \mathrm{LRV}$ ( $\log$ reduction value) is the reduction of virus compared with that of the virus control $* * * \mathrm{P}<0.001$ by one-way ANOVA and Dunnett's post-test, compared with untreated virus control (water). For wells with undetectable virus a value equal to the lower limit of detection was assigned for statistical analyses.

${ }^{\mathrm{c}} \mathrm{CCID} 50 / \mathrm{ml}$ is defined as $50 \%$ cell culture infectious dose per $\mathrm{mL}$.

\section{RESULTS}

The results showing the viral titer and LRV values for SARS-CoV-2 after incubation with a single concentration of nasal spray are shown in Table 2 . Toxicity was observed in the top dilution (1/10). Virus was observed below that dilution and therefore did not affect calculations of viral titer or LRV. After a 25-minute contact time, the nasal spray reduced the levels of virus from 4.2 to $1.7 \log _{10}$ CCID50 per $0.1 \mathrm{~mL}$, a statistically significant reduction of $2.5 \log _{10}$ CCID50. Neutralization controls demonstrated that residual sample did not inhibit viral growth and detection in the endpoint titer assays. Virus controls and positive controls performed as expected.

\section{CONCLUSIONS}

This study demonstrates the strong virucidal effect against SARS-CoV-2 of a nasal spray containing CPM. Given the evidence supporting CPM's antiviral, anti-inflammatory and possible coadjuvant effects with hydroxychloroquine in treating multidrug resistant malaria, together with the results of this in vitro study, we propose two further studies: a randomized placebo-controlled study of intranasally delivered chlorpheniramine in patients with mild to moderate SARS-CoV-2, and a second study aiming to determine the potential antiviral and adjuvant effects of CPM plus hydroxychloroquine, versus chloroquine alone, in hospitalized patients with SARS-CoV-2. 
Given that intranasally delivered CPM has broad antiviral effects with minimal side effects, our study could be extended to test other common respiratory viral infections.

Corresponding Author: gferrer@pulmonary-institute.com

\section{Conflict of Interest}

1. Dr. Ferrer has a patent pending for the intranasal formulation with chlorpheniramine and has received research funding from Xlear, Inc.

2. All other authors report no conflict of interest.

\section{Reference}

1. Zhu N, Zhang D, Wang W, et al; China Novel Coronavirus Investigating and Research Team. A novel coronavirus from patients with pneumonia in China, 2019. N Engl J Med. 2020;382(8):727-733. doi:10.1056/NEJMoa2001017

2. L. Zou et al. 2020. SARS-CoV-2 Viral Load in Upper Respiratory Specimens of Infected Patients. N Engl J Med. 382:1177-1179

3. Xu W, Xia S, Pu J, Wang Q, Li P, Lu L and Jiang S (2018) The Antihistamine Drugs Carbinoxamine Maleate and Chlorpheniramine Maleate Exhibit Potent Antiviral Activity Against a Broad Spectrum of Influenza Viruses. Front. Microbiol. 9:2643. doi: 10.3389/fmicb.2018.02643

4. Sunan N, Phattanapong K; Potentiation of antimalarial drug action by chlorpheniramine against multidrug-resistant Plasmodium falciparum in vitro. Parasitology International 55 (2006) 195-199. doi:10.1016/j.parint.2005.11.058

5. Basco LK, Le Bras J. In vitro reversal of chloroquine resistance with chlorpheniramine in African isolates of Plasmodium falciparum. Jpn J Med Sci Biol 1994;45:59-63

6. Omitowoju GO, Ogundahunsi OAT, Milhous WK, Gerena L, Sowunmi A, Schuster BG., Oduola AMJ. Chlorpheniramine: A resistance reversing agent with potential clinical application. The 41 st annual meeting of The American Society of Tropical Medicine and Hygeine, Seattle, Washington, November 15-19, 1992. Abstract no. 204. Supplement to Am J Trop Med Hyg 1992;47(4) suppl:175

7. Sowunmi A, Oduola AMJ, Ogundahunsi OAT, Salako LA. Comparative efficacy of chloroquine plus chlorpheniramine and pyrimethamine/ sulfadoxine in acute 
uncomplicated falciparum malaria in Nigerian children. Trans R Soc Trop Med Hyg 1998;92:77-81

8. Sowunmi A, Fehintola FA, Ogundahunsi OAT, Oduola AMJ. Comparative efficacy of chloroquine plus chlorpheniramine and halofantrine in acute uncomplicated falciparum malaria in Nigerian children. Trans R Soc Trop Med Hyg 1998;92:441-5

9. Sowunmi A, Oduola AMJ, Ogundahunsi OAT, Falade CO, Gbotosho GO, Salako LA. Enhanced efficacy of chloroquine-chlorpheniramine combination in acute uncomplicated falciparum malaria in children. Trans R Soc Trop Med Hyg 1997;91:63-7

10. Kang-Jye Chou et all. Distribution of antihistamines into the CSF following intranasal

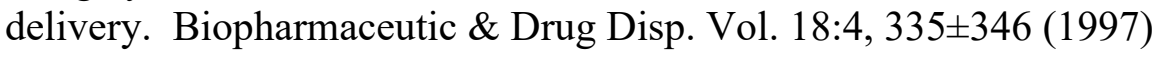

11. B.S.J. van Toor et al. Systemic bioavailability of nasally applied chlorphenamine maleate ( $0.4 \%$ nasal spray) relative to tablets administered perorally. Int J Clin Pharmacol Ther. 39(4):173-178

12. J Kirkegaard et al. Inhibition of histamine-induced nasal symptoms by the $\mathrm{H} 1$ antihistamine chlorpheniramine maleate: Demonstration of topical effect. Br. J. Dis. Chest (1983) 77, 113 